\title{
Ethic Statement Correction: Pull-Through Buddy Wire Technique for Endovascular Thrombectomy in Patients with Acute Ischemic Stroke: Technical Note
}

Pin-Yi Chiang, MD, Yen-Heng Lin, MD, MS, Yu-Cheng Huang, MD, Chung-Wei Lee, MD, PhD

Department of Medical Imaging, National Taiwan University Hospital, Taipei, Taiwan

Correction to: Neurointervention https://doi.org/10.5469/neuroint.2020.00409, published on March, 2021, Neurointervention 2021;16:64-69.

In the originally published version of this article, there were some missing contents in the Ethics Statement section, which should be properly revised as follows:

\section{Before correction:}

\section{Ethics Statement}

The study was approved by the research ethics committee of the National Taiwan University Hospital.

\section{After correction:}

\section{Ethics Statement}

The study was approved by the research ethics committee of the National Taiwan University Hospital. Informed consent for publication was obtained from the patient and/or patient family.

The authors apologize for any inconvenience that it may have caused.

\section{Correspondence to: Chung-Wei Lee, MD, PhD}

Department of Medical Imaging, National Taiwan University Hospital, No. 7, Chung-Shan South Rd, Taipei 100, Taiwan

Tel: +886-2-23123456, Fax: +886-2-23224552, E-mail: rad.chungweilee@gmail.com 